
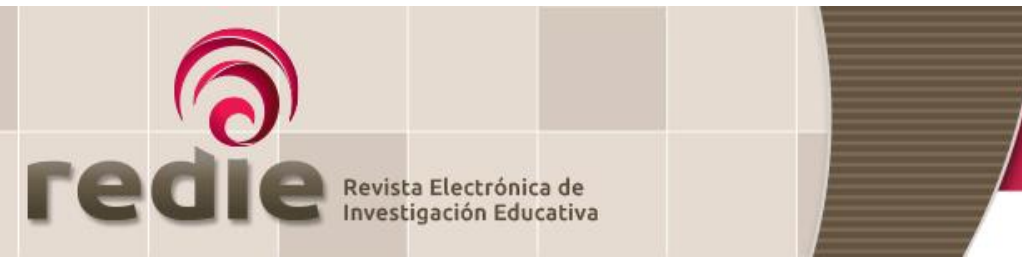

\title{
Relaciones entre contexto de procedencia y logro académico al terminar la primaria ${ }^{1}$
}

\section{Relationship between Context of Origin and Academic Achievement upon Completing Elementary School}

Aldo Bazán-Ramírez (1) https://orcid.org/0000-0001-6260-5097

Eduardo Hernández-Padilla (2) https://orcid.org/0000-0003-4269-9182

César Hernández-Rodríguez (3) https://orcid.org/0000-0003-2121-0798

Eneida Ochoa-Ávila (4) https://orcid.org/0000-0002-6510-8552

(1) Universidad Nacional San Cristóbal de Huamanga

(2) Universidad Autónoma del Estado de Morelos

(3) Comisión Nacional para la Mejora Continua de la Educación

(4) Instituto Tecnológico de Sonora

(Recibido: 25 de marzo de 2018; Aceptado para su publicación: 18 de junio de 2018)

Cómo citar: Bazán-Ramírez, A., Hernández-Padilla, E., Hernández, C. y Ochoa, E. (2020). Relaciones entre contexto de procedencia y logro académico al terminar la primaria. Revista Electrónica de Investigación Educativa, 22, e08, 1-16.

https://doi.org/10.24320/redie.2020.22.e08.2335

\section{Resumen}

Este estudio fue realizado con la finalidad de explicar el efecto que puede tener el contexto de procedencia de los alumnos que concluyeron 6o. grado de primaria en tres indicadores de logro académico: desempeño en una evaluación por aprendizajes esperados en competencias de comprensión lectora y producción de textos, y por aprendizajes esperados en cuatro competencias matemáticas; logro en la prueba ENLACE (Español y Matemáticas); y el promedio de calificación asignado por el profesor de aula al concluir el año escolar. La muestra estuvo conformada por 76 alumnos de escuelas privadas, 103 de escuelas públicas de zona urbana y 123 estudiantes de escuelas indígenas y pública rural. Los resultados indican que: 1) Existen diferencias significativas, de acuerdo con el contexto de procedencia, en los desempeños de los estudiantes en los tres indicadores de logro; 2) Las diferencias de acuerdo con el contexto de procedencia varían en función de las dificultades y características de cada tipo de aprendizaje esperado, y 3) Las relaciones estructurales entre los tres indicadores del logro académico varían en función del contexto de procedencia y de la asignatura evaluada.

Palabras clave: Logro académico, lectura y escritura, matemáticas, contexto de procedencia.

\section{Abstract}

This study was conducted with the goal of explaining the effect that students' context of origin may have on three indicators of academic achievement as they complete elementary school: performance in an assessment of expected learning outcomes in reading and writing skills, and expected learning outcomes

1 Este artículo fue realizado con datos de investigación del Proyecto No. 151981, financiado por el Consejo Nacional de Ciencia y Tecnología de México, en el período 2012-2015. 
in four mathematical skills; achievement in the ENLACE test (in Spanish and mathematics); and the average grade assigned by the class teacher at the end of the school year. The sample was made up of 76 students from private schools, 103 from public schools in urban areas, and 123 students from indigenous schools and a public school in a rural area. The findings show that 1) there are significant differences in student performance in the three indicators of achievement, according to context of origin; 2) the differences based on context of origin vary depending on the difficulties and characteristics of each type of expected learning outcome; and 3 ) the structural relationships between the three indicators of academic achievement vary depending on the context of origin and the subject area evaluated.

Keywords: Academic achievement, reading and writing, mathematics, context.

\section{Introducción}

En este estudio entendemos el término contexto de procedencia de los estudiantes como un conjunto de factores asociados a procesos y resultados de aprendizaje no atribuibles directamente a la situación de aprendizaje en la escuela, e incluye tanto a variables de la comunidad de origen como a variables del hogar y el entorno familiar de origen (Cornejo y Redondo, 2007). Estas variables son denominadas también como factores contextuales -no atribuibles propiamente a la situación de aprendizaje escolar-, pero favorecen o dificultan tanto el aprendizaje (lo que ocurre en el salón de clases o la situación interactiva en la que se da el aprendizaje) como el logro académico (el resultado del aprendizaje, lo que se aprendió). Estos factores influyen de diversas formas promoviendo o inhibiendo el aprendizaje y el rendimiento académico (Ceylan y Kesici, 2017; Emory et al., 2008; González-Pienda, 2003; Steinmayr et al., 2011). El informe Coleman (Coleman et al., 1966) fue un hecho importante en la explicación de la relación entre factores contextuales y el logro académico; según este informe, el contexto de origen de los alumnos explica de forma significativa el logro educativo en pruebas estandarizadas, dejando de lado la contribución de los factores escolares.

Estudios realizados en México y en otros países iberoamericanos con evaluaciones a gran escala de los resultados de aprendizaje (logro educativo), han evidenciado que el contexto de procedencia de los alumnos es un predictor importante del logro, y que un mayor nivel socioeconómico constituye un mejor indicador de logro educativo debido a que los alumnos de los contextos más favorecidos disponen de mayores oportunidades de aprendizaje y de capital cultural (Backhoff, 2011; Backhoff et al., 2010; Blanco, 2008; Carvallo, 2006; Carvallo et al., 2007; Hernández-Padilla y Bazán, 2016; Hernández-Padilla y GonzálezMontesinos, 2011).

Los resultados diferenciados por el contexto de origen de los alumnos mexicanos y de las diferencias por su estatus socioeconómico y cultural, han sido contantes en diversas evaluaciones, tales como las pruebas del Programa para la Evaluación Internacional de los Estudiantes (PISA, por sus siglas en inglés) (Instituto Nacional para la Evaluación de la Educación [INEE], 2013, 2016b); Plan Nacional para la Evaluación de los Aprendizajes (PLANEA) (INEE, 2016d); Exámenes para la Calidad y el Logro Educativo (EXCALE) (INEE, 2016a, 2014); y las evaluaciones Segundo y Tercer Estudio Regional Comparativo y Explicativo (SERCE y TERCE, respectivamente) (INEE, 2016c).

Durante el período 2006 a 2013 la Secretaría de Educación Pública (SEP) aplicó anualmente la prueba censal ENLACE (Evaluación Nacional del Logro Académico en Centros Escolares), de carácter nacional, con el objetivo de evaluar los conocimientos y habilidades en las asignaturas de Español y Matemáticas, en función de los planes y programas de estudio oficiales (ENLACE, 2013). A partir del 2008 se incluyó en cada aplicación una tercera asignatura, la cual rotaba cada año (Ciencias, Formación Cívica y Ética, Historia y Geografía). La última aplicación de la prueba ENLACE en Primaria se realizó en el ciclo escolar 2012-2013, período en el cual se basa esta investigación. 
La prueba constó de 55 y 54 reactivos de opción múltiple para las materias de Español y Matemáticas, respectivamente, y 40 para la asignatura rotativa; la prueba se realizaba durante dos días en los últimos meses del ciclo escolar: 4 sesiones de 45 minutos con descansos de 15 minutos entre cada sesión.

Los resultados de la prueba ENLACE obtenidos entre 2006 y 2013 muestran que los alumnos procedentes de las escuelas particulares obtuvieron resultados significativamente superiores a los alumnos de las escuelas Indígenas, Generales y compensatorias (CONAFE) en los niveles de logro Bueno y Excelente, en los grados 30., 40., 5o. y 60. de Primaria (ENLACE, 2013). De igual forma, diversos estudios realizados en México, utilizando los datos de las evaluaciones del logro académico con la prueba ENLACE, han mostrado que los factores contextuales de procedencia están asociados a bajos desempeños (Alanís et al., 2009; Bautista, 2012; Gutiérrez et al., 2015; Salazar et al., 2010; Tapia y Valenti, 2016).

Si bien es cierto que se han realizados diversas investigaciones que dan cuenta de la relación entre factores de contexto y resultados educativos, el efecto del contexto de procedencia sobre el logro académico en educación básica en México ha sido explicado a partir de las evaluaciones a gran escala, o como análisis secundarios de las bases de datos de tales evaluaciones. Una limitación de este tipo de evaluaciones en el contexto mexicano es que se reportan indicadores globales del logro por asignaturas, o de los niveles de logro cognitivo alcanzados en dichas asignaturas. En ambos casos poco se ha explicado el desempeño en aprendizajes esperados específicos tomando en cuenta el contexto de procedencia de los alumnos. Y poco se ha abordado también la relación entre los promedios de calificaciones obtenidos en el ciclo escolar y los indicadores de logro en evaluaciones a gran escala.

En consecuencia, en este estudio se plantearon tres objetivos:

1. Identificar y explicar el efecto diferencial del contexto de procedencia sobre el logro educativo, en tres diferentes modos de evaluación de logro académico: a) evaluación de aprendizajes esperados por competencias de lectura, producción de textos y matemáticas; b) logro reportado por los puntajes en la prueba ENLACE en Español y Matemáticas de 6o. grado de Primaria; y c) calificación general de 6o. grado asignadas por el profesor de grupo al término del ciclo escolar 2012-2013.

2. Explicar que las diferencias de acuerdo con el contexto de procedencia varían en función de las dificultades y características de cada tipo de aprendizaje esperado, en competencias de comprensión lectora, producción de textos y matemáticas.

3. Establecer relaciones entre índices de logro académico en Español y Matemáticas en 6o. grado (competencias lingüísticas y matemáticas, logro en ENLACE y calificación final por asignatura), por cada contexto de procedencia.

\section{Método}

Se seleccionaron 302 alumnos mediante un muestreo por conveniencia ( $49 \%$ hombres y $51 \%$ mujeres, con una edad promedio de 12 años y una Desviación Estándar (DE) de 0.57), 226 alumnos al inicio de 10. de Secundaria y 76 alumnos al término de 6o. de Primaria.

Los alumnos provenían de siete instituciones escolares del estado de Morelos (México): dos colegios privados ubicados en el municipio de Cuernavaca con 76 estudiantes (Contexto Privado); tres escuelas públicas localizadas en Cuernavaca, con 103 estudiantes (Contexto Público Urbano); y 123 estudiantes procedentes de una escuela indígena localizada en el municipio de Puente de Ixtla, de una escuela indígena del municipio de Temixco y de una escuela pública rural del municipio de Puente de Ixtla (Contexto Ruralindígena).

El criterio de inclusión fue que cada alumno contara con los puntajes en la prueba ENLACE de 60. de Primaria y las calificaciones finales en registro escolar en las asignaturas de Español y Matemáticas en 6o. de primaria. Asimismo, todos los alumnos debían haber completado las evaluaciones de aprendizajes esperados en competencias de Lectura, Producción de Textos y Matemáticas. No se incluyeron estudiantes 
que hubieran repetido grado, que tuvieran alguna discapacidad intelectual, visual o auditiva.

En el caso de los estudiantes que procedían del contexto rural-indígena, aproximadamente el $70 \%$ de la muestra indicó que son bilingües (español y náhuatl) y el 30\% se consideró como monolingüe (español).

\subsection{Instrumentos}

Para la evaluación a pequeña escala de los aprendizajes esperados, por competencias, se utilizaron dos instrumentos independientes, uno para evaluar competencias de comprensión lectora y de producción de textos (Bazán et al., 2013), y otro para evaluar cuatro competencias matemáticas (Ferrari, 2014). Ambos instrumentos, apegados al currículum de 6o. grado de primaria (SEP, 2011), cumplen con criterios de logro en cada pregunta y poseen una estructura de respuestas cualitativamente jerárquicas (tipo rúbrica) en un rango de 0 a 4, distinta a la evaluación con pruebas de respuesta múltiple que tienen una única opción de acierto; en estos instrumentos no existen respuestas incorrectas, sólo valores de logro en rangos cualitativa y cuantitativamente jerarquizados. Por ser instrumentos apegados al currículo escolar ambos cuentan con validez de constructo, de criterio y de jueces expertos. A continuación se describen las características técnicas de los instrumentos.

Instrumento de Comprensión Lectora y Producción de Textos. Evalúa 9 aprendizajes esperados en la competencia Comprensión lectora y 8 en la competencia Producción de textos, con 17 temas de reflexión en común (ver tabla I). La Comprensión lectora se evalúa a partir de textos cortos elaborados de acuerdo con el plan y programa de estudios y similares a los textos incluidos en los libros de texto gratuito de la SEP para 6o. de Primaria. La Producción de textos se evalúa con base en la elaboración escrita de los alumnos (construcción de respuesta) ante instrucciones y demandas señaladas para 8 aprendizajes esperados de acuerdo con el plan y programas de estudio y el libro de texto para 6o. de Primaria.

En la competencia Comprensión lectora se obtuvo un buen índice de consistencia interna (Alfa de Cronbach de 0.92); asimismo, cada uno de los 9 aprendizajes esperados evaluados en esta competencia obtuvieron índices de consistencia entre 0.78 y 0.92 . Mediante el análisis factorial confirmatorio se obtuvo buena validez convergente y divergente de constructo, y buena bondad de ajuste entre el modelo hipotetizado y el modelo observado ( $x^{2}=45.67, p=0.01 ; C F I=.97$ y RMSA=0.05), confirmándose los 9 constructos de aprendizajes esperados de Comprensión lectora.

En Producción de textos el índice de consistencia interna general con Alfa de Cronbach fue de 0.88, en tanto que los índices de consistencia interna por aprendizajes esperados variaron entre 0.86 y 0.94. De igual forma, se obtuvo validez convergente y divergente de constructos con aceptable bondad de ajuste $\left(\mathrm{x}^{2}=\right.$ 72.30, $\mathrm{P}=0.00, \mathrm{CFI}=.92$ y RMSA $=0.09$ ), confirmando de esta manera la evaluación de producción de textos con 8 aprendizajes esperados. 
Tabla I. Estructura del instrumento de evaluación del desempeño de Comprensión lectora y Producción de textos en 6o. grado de Primaria

\begin{tabular}{|c|c|c|c|}
\hline \multicolumn{2}{|r|}{ Aprendizajes Esperados } & Competencia & Ejercicios \\
\hline $\begin{array}{l}\checkmark \\
\checkmark \\
\checkmark \\
\checkmark \\
\checkmark \\
\checkmark \\
\checkmark \\
\checkmark \\
\checkmark\end{array}$ & $\begin{array}{l}\text { Identificar información específica en } \\
\text { el contenido del recuento histórico } \\
\text { (Información explícita). } \\
\text { Inferir fechas y lugares a partir de } \\
\text { textos narrativos de hechos reales } \\
\text { (Información implícita). } \\
\text { Identifica errores en la ortografía de } \\
\text { los textos. } \\
\text { Comprender metáforas y otras figuras } \\
\text { usadas en los cuentos. } \\
\text { Identificar las características de los } \\
\text { cuentos de misterio o terror (la } \\
\text { estructura, el estilo, los personajes y } \\
\text { el escenario). } \\
\text { Infiere el significado de palabras en } \\
\text { lengua indígena. } \\
\text { Identificar las secciones del periódico. } \\
\text { Reconoce el lenguaje figurado y su } \\
\text { función para evocar emociones. } \\
\text { Reconoce las figuras literarias } \\
\text { utilizadas en la poesía }\end{array}$ & $\begin{array}{l}\text { Comprensión } \\
\text { lectora }\end{array}$ & $\begin{array}{l}\text { a) Emiliano Zapata y la revolución } \\
\text { agraria del sur } \\
\text { b) Tras los pasos de Hidalgo } \\
\text { c) Un joven que viajó lejos para } \\
\text { saber lo que era el miedo } \\
\text { d) El gato negro } \\
\text { e) Dentro de mil años } \\
\text { f) El cuento náhuatl/yaqui } \\
\text { g) El periódico } \\
\text { h) Emociones } \\
\text { i) Poemas }\end{array}$ \\
\hline $\begin{array}{l}\checkmark \\
\checkmark \\
\checkmark \\
\checkmark \\
\checkmark \\
\checkmark \\
\checkmark \\
\checkmark\end{array}$ & $\begin{array}{l}\text { Redacta preguntas para elaborar una } \\
\text { entrevista. } \\
\text { Elaborar una carta de opinión, a partir } \\
\text { de una noticia y una carta informal. } \\
\text { Convierte el discurso directo a } \\
\text { discurso indirecto. } \\
\text { Escribir cartas personales a familiares } \\
\text { o amigos. } \\
\text { Describir a las personas de forma } \\
\text { precisa. } \\
\text { Narra eventos de manera breve. } \\
\text { Elaborar una biografía. } \\
\text { Elaborar una autobiografía. }\end{array}$ & $\begin{array}{l}\text { Producción de } \\
\text { textos }\end{array}$ & $\begin{array}{l}\text { a) La entrevista } \\
\text { b) La carta de opinión } \\
\text { c) El discurso } \\
\text { d) A mi querido amig@ } \\
\text { e) El personaje } \\
\text { f) Posada navideña } \\
\text { g) Biografía } \\
\text { h) Autobiografía }\end{array}$ \\
\hline
\end{tabular}

Instrumento de Matemáticas. En la tabla II se presenta la estructura del instrumento de evaluación de competencia matemática que incluye las 4 competencias establecidas para desarrollar en 6o. de Primaria; incluye 12 ejercicios o series de actividades de evaluación, 3 por cada competencia. Cada ejercicio o serie incluye 2 actividades de evaluación orientadas a aprendizajes de operaciones matemáticas y 2 actividades orientadas a los aprendizajes esperados de reflexión y argumentación de las respuestas a los ejercicios matemáticos.

El índice de consistencia interna valorado mediante el coeficiente Alfa de Cronbach para todo instrumento para evaluar la competencia matemática fue de 0.88. Tomando cada una de las cuatro competencias, los índices de consistencia interna fluctuaron entre 0.69 y 0.74. El análisis factorial confirmatorio (AFC) de las 4 competencias y 2 aprendizajes esperados por cada competencia, mostró validez convergente y divergente de constructos, con una buena bondad de ajuste $\left(x^{2}=43.35 ; p=0.00 ; \mathrm{CFI}=0.98 ;\right.$ RMSEA $\left.=0.07\right)$. 
Tabla II. Estructura del Instrumento de evaluación de Matemáticas para 6o. grado de primaria

\begin{tabular}{|c|c|c|}
\hline Aprendizajes Esperados & Competencias & Ejercicio \\
\hline $\begin{array}{l}\text { - Elige representación gráfica } \\
\text { - Explica los elementos o contenidos }\end{array}$ & $\begin{array}{l}\text { Comunicar información } \\
\text { matemática }\end{array}$ & $\begin{array}{l}\text { a) Equipo de basquetbol } \\
\text { b) Las caricaturas } \\
\text { c) Cuernavaca }\end{array}$ \\
\hline $\begin{array}{l}\text { - Expresa promedios y resultados } \\
\text { - Argumenta procedimientos y resultados. }\end{array}$ & $\begin{array}{l}\text { Validar procedimientos } \\
\text { y resultados }\end{array}$ & $\begin{array}{l}\text { a) ¿Sabías qué? Pirámide de } \\
\text { Kukúlkan } \\
\text { b) El principito } \\
\text { c) Canicas }\end{array}$ \\
\hline $\begin{array}{l}\text { - Identifica y resuelve problemas. } \\
\text { - Probar eficacia de un procedimiento } \\
\text { cambiando variables. }\end{array}$ & $\begin{array}{l}\text { Resolver problemas de } \\
\text { manera autónoma }\end{array}$ & $\begin{array}{l}\text { Cajas y cubos } \\
\text { ¿Sabías qué? Torre Khalifa } \\
\text { Algo de historia }\end{array}$ \\
\hline $\begin{array}{l}\text { - Elegir operaciones e indica procedimientos } \\
\text { resolviendo el problema. } \\
\text { - Usar eficientemente procedimientos al } \\
\text { efectuar cálculos. }\end{array}$ & $\begin{array}{l}\text { Manejar técnicas } \\
\text { eficientemente }\end{array}$ & $\begin{array}{l}\text { a) El cofre secreto } \\
\text { b) Se me antoja una rebanada } \\
\text { de pastel } \\
\text { c) Chicharito }\end{array}$ \\
\hline
\end{tabular}

\subsection{Procedimiento}

El estudio fue realizado de junio a octubre de 2013. En primer lugar, una vez seleccionadas las secciones (grupos) participantes, los investigadores tuvieron una reunión informativa con los alumnos y el profesor de cada grupo escolar, en el mismo salón de clases, para explicarles los objetivos del estudio. Asimismo, se les explicó que los resultados eran para mostrar lo que ellos sabían en las asignaturas de Español y Matemáticas, pero que no tenían efectos en la calificación en el registro escolar, se les dijo también que una vez concluido el estudio los investigadores regresarían a presentar los resultados del grupo y una retroalimentación individual de forma verbal y visual, lo cual se cumplió.

Al final de la sesión informativa a cada alumno se le entregó un formato de asentimiento para que los que quisieran participar lo firmaran o escribieran su nombre. A los padres de los niños que aceptaron participar en el estudio se les envío un sobre con una explicación corta sobre los objetivos del estudio y un formato de consentimiento informado, el cual devolvieron por medio de sus hijos.

En las tres sesiones siguientes se realizaron las evaluaciones en los mismos salones de clase, con una duración promedio de 60 a 90 minutos. El primer día fue de Comprensión lectora, el segundo de Producción de textos y el tercero de Matemáticas. En todos los casos, y antes de distribuir los cuadernillos de evaluación, los aplicadores daban instrucciones generales, realizaban un ejemplo y permanecían en el salón durante toda la sesión.

La aplicación de las evaluaciones de aprendizajes en las competencias de Comprensión lectora y Producción de textos y Matemáticas de 6o. grado de primaria fue realizada en dos períodos diferentes: 1) en junio de 2013, cuando los alumnos cursaban el último mes del 6o. grado de primaria (ciclo escolar 2012-2013); y 2) en agosto, cuando los alumnos iniciaban el 1er. grado de educación secundaria (primer mes del ciclo escolar 2013-2014).

A partir de septiembre de 2013 se obtuvieron en cada una de las escuelas participantes las calificaciones finales de cada alumno al finalizar 6o. grado (la nota o calificación general, y las calificaciones en Español y Matemáticas). También se obtuvieron los puntajes en la prueba ENLACE en la página web de la SEP, utilizando la Clave Única de Registro de Población (CURP) de cada alumno.

\subsection{Análisis de los datos}

Рara la comparación de los resultados de aprendizaje o logro académico dependiendo del contexto de procedencia se construyeron índices de cada aprendizaje esperado por cada competencia evaluada. Posteriormente, con los índices de aprendizajes esperados, el promedio de calificaciones otorgadas por el profesor de grupo y los índices de logro en la prueba ENLACE (puntaje en Español y en Matemáticas) fueron 
realizados análisis comparativos de los desempeños académicos de los alumnos por contexto de procedencia y por aprendizajes esperados, y mediante el análisis de varianza se evaluó si existían diferencias significativas entre los estudiantes de diferentes contextos en los indicadores de desempeño académico; para estos análisis se empleó el paquete estadístico sPSS versión 23.

Además, para cada asignatura evaluada (Español y Matemáticas) se probó un modelo hipotético de relaciones estructurales en cada contexto de procedencia (privado, urbano público y rural-indígena), entre competencias lingüísticas y matemáticas, logro en la prueba ENLACE (en Español y Matemáticas), y calificación final en 6o. grado en Español y Matemáticas. Para el modelamiento de ecuaciones estructurales del modelo general y de los correspondientes a cada contexto se usó el software EQS 6.2.

\section{Resultados}

En la tabla III se muestran los resultados de análisis de varianza de un factor de las calificaciones escolares y de los puntajes obtenidos en la prueba ENLACE. En ambos casos se observan diferencias estadísticamente significativas entre los alumnos de los tres contextos, a favor de los alumnos del contexto privado.

Tabla III. Análisis de varianza de un factor de las calificaciones escolares y los puntajes obtenidos en la prueba ENLACE

\begin{tabular}{l|c|c|c|c|c}
\hline \multirow{2}{*}{ Desempeño } & \multicolumn{3}{|c|}{ Medias } & \multirow{2}{*}{$F$} & \multirow{2}{*}{$\boldsymbol{P}$} \\
\cline { 2 - 5 } & Privado & Urbano & $\begin{array}{c}\text { Rural } \\
\text { Indígena }\end{array}$ & F & \\
\hline Calificación Escolar general & 8.40 & 8.51 & 8.21 & 4.252 & $<.050$ \\
\hline ENLACE español & 711 & 510 & 459 & 133.565 & $<.000$ \\
\hline ENLACE Matemáticas & 702 & 540 & 527 & 58.478 & $<.000$ \\
\hline
\end{tabular}

En las tablas IV, V y VI se presentan los resultados del desempeño en la evaluación por aprendizajes esperados en competencias lingüísticas (relacionadas con la asignatura de Español) y en competencias matemáticas (relacionadas con la asignatura de Matemáticas), en términos de proporción de aciertos según el contexto de procedencia (en un rango de 0 a 100). Las diferencias entre los contextos de procedencia fueron estimadas mediante el análisis de varianza de un factor.

La tabla IV presenta la comparación de medias entre los 3 contextos de procedencia en los 9 aprendizajes esperados de la competencia Comprensión lectora. Además de los indicadores de significatividad de tales diferencias se pueden observar los promedios de proporción de aciertos logrados en cada contexto por cada aprendizaje esperado, siendo favorables en todos los casos para los alumnos del contexto privado, mientras que los alumnos del contexto rural-indígena obtuvieron los índices más bajos de proporción de aciertos en todos los aprendizajes esperados. 
Tabla IV. Análisis de varianza de un factor de los aprendizajes esperados en Comprensión lectora por contexto de procedencia

\begin{tabular}{l|c|c|c|c|c}
\hline \multicolumn{1}{c|}{ Aprendizaje esperado } & \multicolumn{3}{c|}{ Medias } & F & $P$ \\
\cline { 2 - 6 } & Privado & Urbano & $\begin{array}{c}\text { Rural- } \\
\text { indígena }\end{array}$ & & \\
\hline Inferir información explícita & 49.72 & 35.74 & 27.65 & 26.741 & $<.001$ \\
Inferir información implícita & 69.32 & 36.80 & 32.29 & 62.434 & $<.000$ \\
Comprender metáforas encontradas en los cuentos & 40.48 & 18.33 & 13.91 & 20.937 & $<.000$ \\
Identificar características de los cuentos & 61.08 & 40.45 & 26.23 & 60.011 & $<.000$ \\
Identificar errores ortográficos & 76.14 & 40.70 & 24.51 & 62.657 & $<.000$ \\
Inferir palabras de Náhuatl o Yaki & 63.92 & 42.77 & 34.93 & 21.716 & $<.000$ \\
Identifica secciones del periódico & 40.77 & 28.58 & 13.60 & 29.868 & $<.000$ \\
Reconoce el lenguaje figurado y su función para & 50.43 & 23.38 & 12.68 & 70.746 & $<.000$ \\
evocar emociones & 6.25 & 4.63 & 1.79 & 4.790 & $<.010$ \\
\hline Reconoce figuras literarias en poesía & & &
\end{tabular}

La tabla V presenta la comparación de medias entre los contextos de procedencia en los ocho aprendizajes esperados de la competencia Producción de Textos, la cual muestra diferencias significativas a favor de los alumnos del contexto privado, con índices de proporción de aciertos más altos en alumnos del contexto privado y con proporción de acierto más bajos en alumnos del contexto rural - indígena.

Tabla V. Análisis de varianza de un factor de los aprendizajes esperados en Producción de textos, por contexto de procedencia

\begin{tabular}{l|c|c|c|c|c}
\hline \multirow{2}{*}{\multicolumn{1}{c|}{ Aprendizaje esperado }} & \multicolumn{3}{c|}{ Medias } & F & $P$ \\
\cline { 2 - 6 } & Privado & Urbano & $\begin{array}{c}\text { Rural- } \\
\text { indígena }\end{array}$ & & \\
\hline Redactar preguntas para una entrevista & 87.50 & 77.42 & 60.85 & 21.203 & $<.000$ \\
Redactar una carta de opinión & 48.44 & 20.79 & 10.23 & 50.351 & $<.000$ \\
\hline Convierte discurso directo en indirecto & 18.94 & 16.29 & 6.73 & 13.608 & $<.000$ \\
Elabora una carta personal & 69.32 & 35.11 & 12.32 & 65.842 & $<.000$ \\
\hline Describir a un personaje de una manera precisa & 65.20 & 27.67 & 5.85 & 116.981 & $<.000$ \\
Narra eventos de una manera breve & 47.02 & 19.66 & 5.76 & 70.1 & $<.000$ \\
Elaborar autobiografía & 54.83 & 34.83 & 22.55 & 20.718 & $<.000$ \\
Elaborar biografía & 53.50 & 33.87 & 21.02 & 36.264 & $<.000$ \\
\hline
\end{tabular}

De acuerdo con las tablas IV y V, de los 17 aprendizajes esperados derivados de la asignatura de Español en 6o. grado de primaria que fueron incluidos en este estudio para evaluar competencias de Comprensión lectora y Producción de textos, los alumnos de escuelas privadas obtuvieron porcentajes de acierto del 60\% o más en 7 de ellos; 4 en Comprensión lectora (Identificar errores ortográficos = 76\%, Inferir información implícita = 69\%, Inferir palabras de Náhuatl o Yaki =64\%, e Identificar características de los cuentos =61\%) y 3 en producción de textos (Redactar preguntas para una entrevista $=87 \%$, Elabora una carta personal = $69 \%$, y Describir a un personaje de una manera precisa $=65 \%$ ). En contraste, tanto los alumnos que proceden de escuelas públicas urbanas como los alumnos de las escuelas rurales y/o indígena obtuvieron un porcentaje mayor al 60\% de aciertos sólo en uno de los 17 aprendizajes esperados evaluados: en redactar preguntas para una entrevista en la competencia Producción de textos (77\% y 61\%, respectivamente).

Por último, la tabla VI muestra la comparación de medias entre los tres contextos educativos, en cada aprendizaje esperado evaluado en las cuatro competencias matemáticas. En todos los casos se obtuvieron diferencias estadísticamente significativas a favor de los alumnos del contexto privado. La tabla muestra que sólo en uno de los 8 aprendizajes esperados (Elige representación gráfica) los alumnos de los tres contextos obtuvieron más del $70 \%$ de aciertos; en tres aprendizajes esperados (Expresa promedios y resultados, Identifica y resuelve problemas y Elige operaciones e indica procedimientos resolviendo el 
problema) se alcanzó un porcentaje de aciertos entre $52 \%$ y 54\%, pero sólo en el contexto privado; es decir, los alumnos de escuelas públicas del contexto urbano y los del contexto rural-indígena obtuvieron porcentajes de acierto inferior al 50\% en 7 de los 8 aprendizajes esperados, mientras que los alumnos del contexto privado lograron menos del 50\% de aciertos, sólo en 4 de los 8 aprendizajes esperados.

Tabla VI. Análisis de varianza de un factor de los aprendizajes esperados de las competencias matemáticas y medias de proporción de acierto por contexto

\begin{tabular}{|c|c|c|c|c|c|}
\hline \multirow[b]{2}{*}{ Aprendizaje esperado } & \multicolumn{3}{|c|}{ Medias } & \multirow[b]{2}{*}{$\mathrm{F}$} & \multirow[b]{2}{*}{$p$} \\
\hline & Privado & Urbano & $\begin{array}{c}\text { Rural- } \\
\text { indígena }\end{array}$ & & \\
\hline Elige representación gráfica & 85.63 & 78.86 & 70.79 & 13358.00 & $<.000$ \\
\hline $\begin{array}{l}\text { Menciona o explica los elementos o } \\
\text { contenidos }\end{array}$ & 30.17 & 21.38 & 18.54 & 15715.00 & $<.000$ \\
\hline Expresa promedios y resultados & 51.92 & 43.19 & 40.57 & 7906.00 & $<.000$ \\
\hline Argumenta procedimientos y resultados & 31.23 & 21.33 & 16.33 & 18452.00 & $<.000$ \\
\hline Identifica y resuelve problemas & 53.64 & 36.05 & 31.46 & 29888.00 & $<.000$ \\
\hline $\begin{array}{l}\text { Probar eficacia de un procedimiento } \\
\text { cambiando variables }\end{array}$ & 41.95 & 32.52 & 29.51 & 12144.00 & $<.000$ \\
\hline $\begin{array}{l}\text { Elegir operaciones e indica procedimientos } \\
\text { resolviendo el problema }\end{array}$ & 54.21 & 39.38 & 36.10 & 18178.00 & $<.000$ \\
\hline $\begin{array}{l}\text { Usar eficientemente procedimientos al } \\
\text { efectuar cálculos }\end{array}$ & 20.40 & 18.32 & 16.92 & 3435.00 & $<0.050$ \\
\hline
\end{tabular}

Los datos de la tabla muestran también que en todos los aprendizajes esperados evaluados son los alumnos del contexto privado los que obtienen mayores porcentajes de aciertos, y los alumnos del contexto ruralindígena los que obtienen los porcentajes más bajos. Asimismo, en el segundo aprendizaje esperado en cada una de las cuatro competencias matemáticas, los alumnos de los tres contextos de procedencia obtuvieron porcentajes más bajos en contraste con lo que obtienen en el primer aprendizaje esperado de cada competencia.

\subsection{Relaciones estructurales entre indicadores de logro por contexto}

En la figura 1 se presentan los tres modelos estructurales resultantes de relaciones entre tres indicadores de logro académico correspondientes a la asignatura de Español 6o. grado (contexto privado, contexto urbano pública y contexto rural-indígena). Los tres modelos incluyeron una variable latente denominada competencias lingüísticas (y sus indicadores de Comprensión lectora y Producción de textos), una variable latente denominada ENLACE Español (conformado por los índices nivel de logro y puntaje obtenido en Español), y una variable manifiesta (Calificación final obtenida en Español durante todo el año escolar).

Según se muestra en la figura 1 , en los tres contextos de procedencia se obtuvieron modelos con buena bondad de ajuste: $\mathrm{P}=0.51$ en el contexto privado, 0.71 en el contexto urbano y 0.36 en el contexto ruralindígena; CFI = 1.0 en los tres modelos; RMSEA $=0.00$ en el contexto privado y en el contexto urbano, y 0.03 en el contexto rural-indígena.

Las correlaciones (covariaciones) entre los tres indicadores de logro son más bajas en el contexto privado y van de 0.47 a 0.58 , mientras que en el contexto urbano varía entre 0.65 a 0.72 , y en el contexto ruralindígena van de 0.62 a 0.72. La relación entre la calificación en Español y el logro en ENLACE Español es más alta en los contextos urbano y rural-indígena (0.72), en comparación con el contexto privado (0.52); La relación entre las variables latentes competencias lingüísticas y logro en ENLACE Español es más alta en el contexto urbano (0.70), en contraste con el contexto rural-indígena (0.62) y el contexto privado (0.58). Asimismo, la relación entre la calificación anual en Español y el logro en ENLACE Español es más alta en el contexto urbano y rural-indígena (0.72) que en el contexto privado (0.52). 

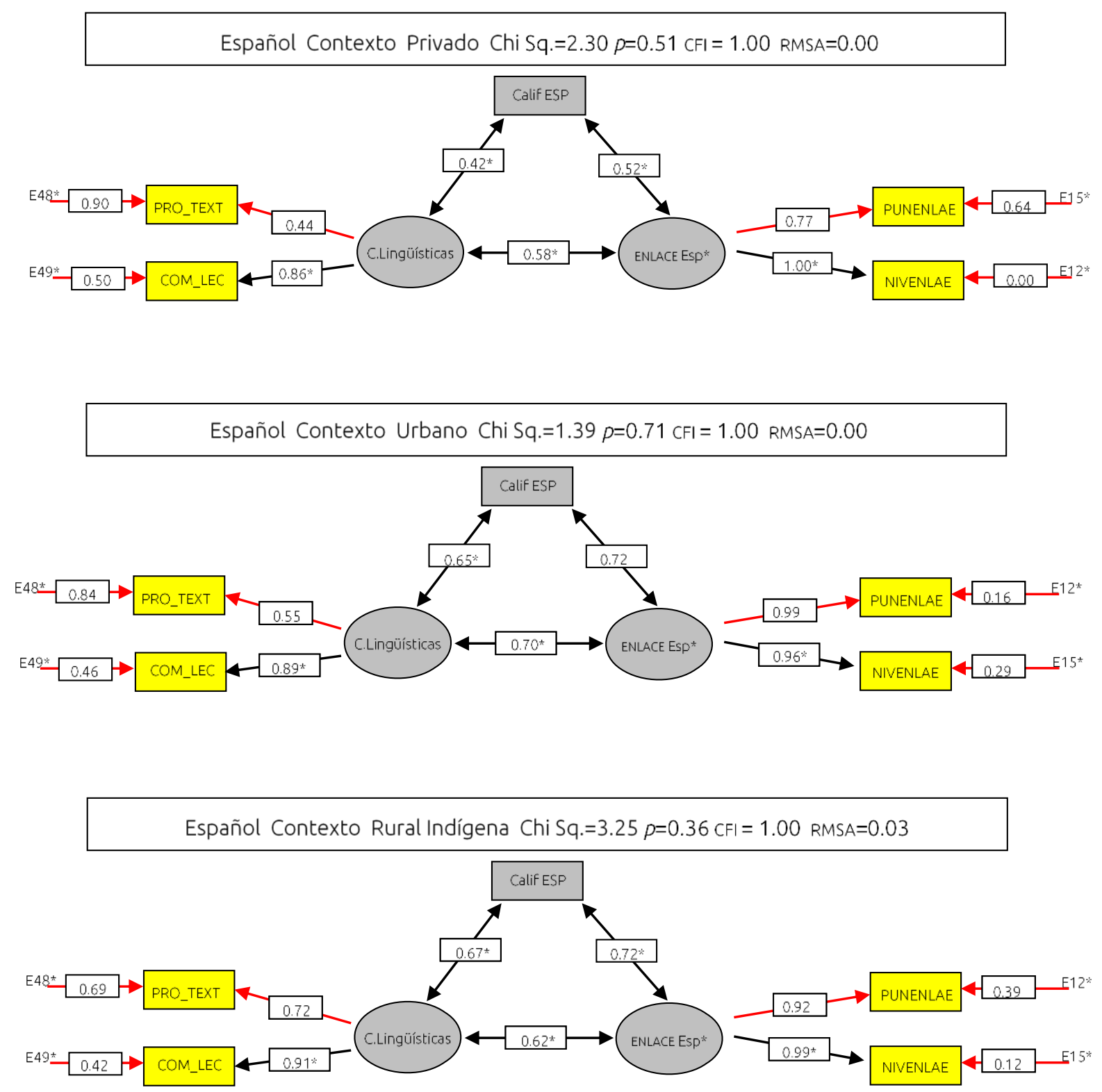

Figura 1. Relaciones entre indicadores de logro en Español según el contexto de procedencia

En la figura 2 se presentan los tres modelos estructurales resultantes de relaciones entre indicadores de logro académico correspondientes a la asignatura de Matemáticas de 6o. grado (contexto privado, contexto urbano pública y contexto rural-indígena). Los tres modelos incluyeron una variable latente denominada competencia matemática conformado por cuatro índices (Comunicar información matemática, Validar procedimientos y resultados, Resolver problemas de manera autónoma y Manejar 
técnicas eficientemente), una variable latente denominada ENLACE Matemáticas (conformado por los índices nivel de logro y puntaje obtenido en Matemáticas), y una variable manifiesta (Calificación final obtenida en Matemáticas en el año escolar).
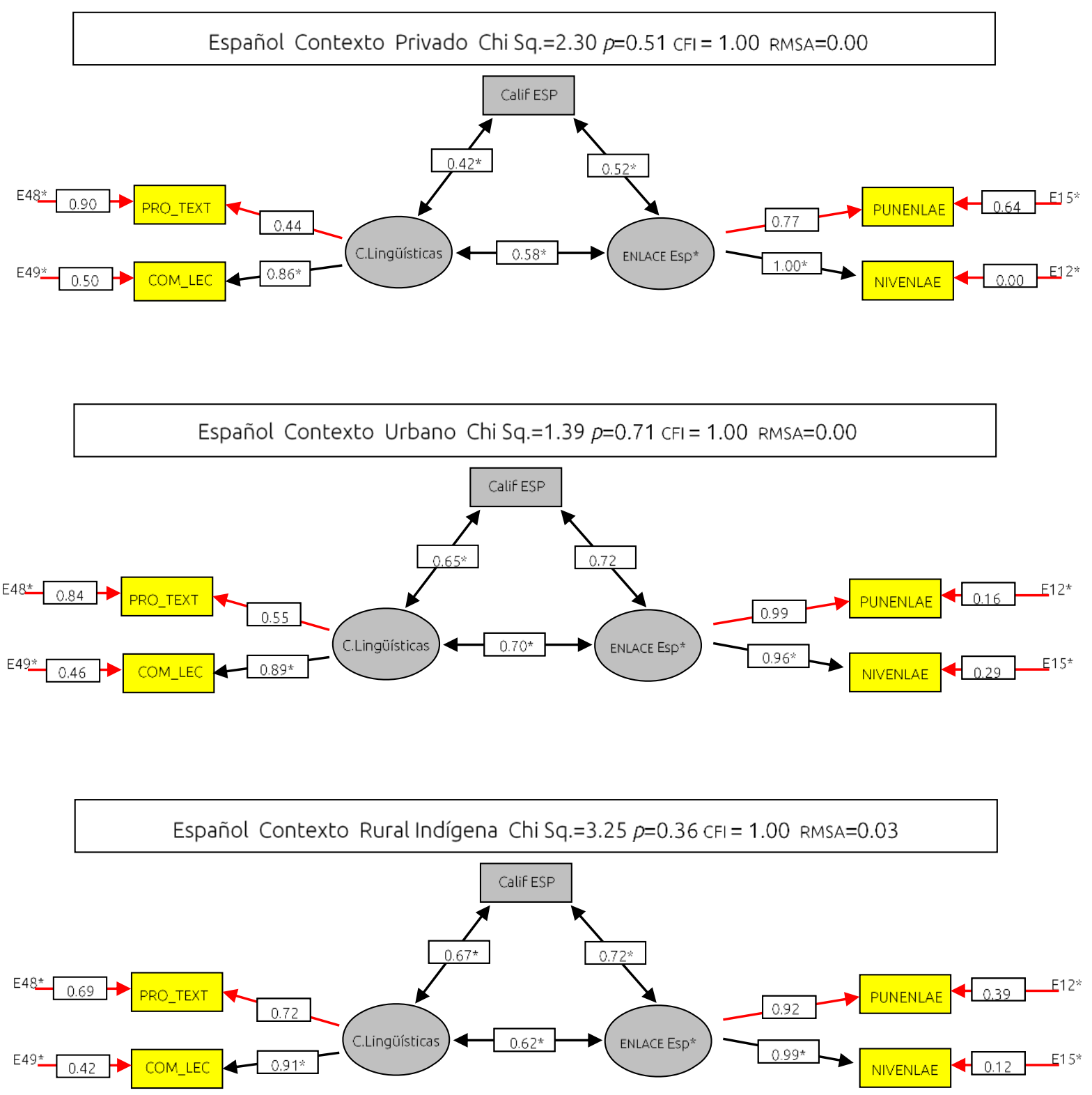

Figura 2. Relaciones entre indicadores de logro en Matemáticas según el contexto de procedencia

Los modelos obtenidos para el contexto privado y para el rural-indígena presentaron excelentes indicadores de bondad de ajuste respecto al modelo teórico ( $P=0.65$ y $0.12 ; C F I=1.00$ y $0.99 ;$ RMSEA $=0.00$ y 0.07 ), y en el contexto urbano se obtuvo una buena bondad de ajuste entre el modelo obtenido y el 
modelo teórico ( $\mathrm{P}=0.06 ; \mathrm{CFI}=1)$, pero con un indicador de RMSEA alto $(0.10)$ que indica un ajuste moderado o mediocre.

En cuanto a las relaciones entre los indicadores de logro o desempeño en matemáticas, en el contexto rural-indígena se obtuvieron mejores índices de covariación, seguido por el contexto privado. En los tres contextos, los mejores índices de relación se dan entre la calificación anual en Matemáticas y el logro en ENLACE Matemáticas: 0.74 en el rural-indígena y 0.64 tanto en el contexto privado como en el contexto urbano. Por otra parte, la relación entre el logro en evaluación de competencias matemáticas y el logro en la prueba ENLACE Matemáticas, también es más alta en el contexto rural-indígena (0.59) en contraste con el contexto privado (0.52) y el urbano (0.51). Por último, la relación entre la calificación en Matemáticas y logro en evaluación por competencias y aprendizajes esperados en Matemáticas es más baja en el contexto urbano (0.35) y mejor en el contexto rural-indígena (0.60), seguido por el contexto privado (0.50).

\section{Discusión}

Los análisis preliminares mostraron que tanto en la evaluación independiente por aprendizajes esperados en competencias lingüísticas y matemáticas, así como en el desempeño en la prueba ENLACE Español y Matemáticas, los alumnos procedentes de las escuelas privadas obtuvieron indicadores de desempeño más altos, seguido por los alumnos de escuelas públicas urbana y en último lugar, por los alumnos de las escuelas del contexto rural-indígena. De igual manera, hubo diferencias significativas por contexto de procedencia en el promedio general de calificaciones otorgadas por el profesor de grupo en las asignaturas Español y Matemáticas, a favor de los alumnos de las escuelas privadas.

Estos datos concuerdan con los antecedentes de las evaluaciones con la prueba ENLACE, los cuales habían mostrado que el logro académico depende de la modalidad o tipo de la escuela de procedencia, con clara ventaja para los alumnos de las escuelas particulares y esventaja para los alumnos de escuelas indígenas y pública general rural (ENLACE, 2013; Tapia y Valenti, 2016). La modalidad o tipo de escuela está relacionada con aspectos socioeconómicos, demográficos y culturales de origen, entre ellos, el entorno comunal, familiar y escolar, que en conjunto constituyen lo que se ha denominado contexto de procedencia o contexto de origen de los estudiantes (Cornejo y Redondo, 2007; González-Pienda, 2003).

Los datos del presente estudio reflejan que el contexto de procedencia parece influir en los tres indicadores de desempeño o logro académico, tanto en la asignatura de Español como en Matemáticas, también evidencian que existe una desigualdad entre los resultados de aprendizaje de acuerdo con el contexto de origen de los alumnos, de sus escuelas y familias. Estos datos revelan la existencia de diferencias por la modalidad escolar, evidencia que es acorde con resultados mostrados en diversas pruebas estandarizadas, tales como, PISA (INEE, 2016b, 2013), Excale (INEE, 2016a, 2014), TERCE (INEE, 2016c).

Estos datos también parecen reflejar las diferencias en las oportunidades educativas, el acceso, los procesos y efectos educativos, de acuerdo con el contexto de origen de los alumnos. Otros estudios también han resaltado que las diferencias de resultados en evaluaciones de logro académico están en función del contexto de procedencia y están estrechamente relacionados con los niveles socioeconómicos, culturales e índices de marginalidad, tanto de las familias de origen como de las escuelas de procedencia (Alanís et al., 2009; Backhoff, 2011; Bautista, 2012; Gutiérrez et al., 2015; Salazar et al., 2010).

Por otra parte, además de proporcionar datos sobre las diferencias de logro educativo según el contexto de procedencia, las evaluaciones por aprendizajes esperados permiten identificar con mayor precisión el grado en que se logró un objetivo instruccional dependiendo del contexto de procedencia.

Los resultados por aprendizajes esperados en este estudio evidencian que independientemente del contexto socioeconómico y cultural de las familias de origen, existen ciertos aprendizajes al término del sexto de primaria, en los que los alumnos de los tres contextos presentan de manera similar dificultades, como es el caso de Reconocer figuras literarias en poesía (un aprendizaje de la competencia comprensión lectora), en el cual en ninguno de los tres contextos se logró más del 7 \% de proporción de aciertos. De igual manera, en el aprendizaje esperado Convierte discurso directo en indirecto, en la competencia 
producción de textos, los alumnos de los tres contextos obtuvieron el promedio de aciertos más bajos al escribir textos.

Asimismo, en las competencias matemáticas los resultados también reflejaron que independientemente del contexto o modalidad escolar, hay una tendencia a que los alumnos de los tres contextos de procedencia obtengan desempeños aún más bajos en los aprendizajes esperados que evalúan la reflexión y la argumentación en matemáticas, comparado con su desempeño en aprendizajes orientados a operaciones y problemas matemáticos. Es decir, estos estudiantes en general, pasan a la secundaria no solamente con indicadores de conocimientos matemáticos en un nivel moderado o bajo, sino que muestran también un desarrollo insuficiente de alfabetización matemática, puesto que en los tres contextos tuvieron mayor dificultad para explicar los elementos o contenidos de un procedimiento matemático, argumentar sus procedimientos y consecuencias, probar algún procedimiento cambiando variables y usar procedimientos variados al efectuar cálculos.

En los modelos de relaciones estructurales entre los tres indicadores de desempeño o logro académico, los resultados fueron diferentes de acuerdo con el contexto de procedencia y según la asignatura. En Español, las correlaciones más altas fueron en el contexto urbano y en el contexto rural-indígena, y las correlaciones más bajas fueron en el contexto privado, en cambio en Matemáticas, las correlaciones más altas fueron en el contexto rural - indígena, seguido por el contexto privado. Por otra parte, los datos de este estudio muestran que la relación entre indicadores de logro en evaluaciones sistemáticas (de aprendizajes esperado en competencias lingüísticas y matemáticas y el logro en ENLACE), en Español es más alta en el contexto urbano público y la más baja se dio en el contexto privado. En Matemáticas, las relaciones más altas se dieron en el contexto rural-indígena.

Estas diferencias pueden deberse a divergencias en las condiciones educativas y socio culturales propias de cada contexto de procedencia. En las escuelas públicas en el contexto urbano y rural probablemente hay mayor apego en la enseñanza y trabajo en el aula a los planes y programas de la SEP y a los libros de texto gratuito, especialmente en las zonas rurales donde hay menor disposición y acceso a materiales complementarios para el aprendizaje escolar. Por el contrario, en las escuelas privadas la enseñanza no se apega estrictamente a los planes y programas de la SEP, sino que además de ello, utilizan otros libros y materiales, y en consecuencia, los alumnos trabajan con mayor número de actividades y variedad de materiales educativos, en contraste con los alumnos de escuelas públicas. Por otra parte, en el contexto privado el promedio asignado al alumno no solo certifica que este ha aprendido los contenidos curriculares, sino, que ha desarrollado conocimientos y habilidades más amplios, que posteriormente le permiten obtener mejores calificaciones en las evaluaciones de logro, por ejemplo, la prueba ENLACE. Una posible explicación de los puntajes altos en la prueba ENLACE de los estudiantes de escuelas privadas se deba a un mayor capital cultural de las familias de origen y las condiciones y acceso a materiales, métodos y programas complementarios en el aprendizaje y uso de las matemáticas, en el contexto rural el aprendizaje y desarrollo de competencias matemáticas son determinados mayormente por los contenidos curriculares y los libros de texto gratuito que la secretaría de educación les proporciona.

El alto índice de relación entre logro de los aprendizajes curriculares esperados y el logro en la prueba ENLACE en el contexto rural-indígena, puede estar sugiriendo para este contexto, la importancia que tiene la enseñanza y los materiales educativos complementarios orientado a competencias y el logro de aprendizajes esperados, apegados tanto al enfoque como a la operación didáctica de los planes y programas de estudio, para proporcionar a estos estudiantes las oportunidades en el salón de clases propicias para su aprendizaje (Jensen et al., 2015), y la posibilidad de resultados prometedores de los enfoques centrados en competencias y de aprendizajes esperados, en especial en alumnos de contextos rurales (Bazán et al., 2013).

Otra explicación plausible es que el aprendizaje de Matemáticas depende en gran medida de lo que se enseña en la escuela y no tanto del contexto sociocultural, como en el caso de Español, por lo tanto, las diferencias entre contextos no son atribuibles directamente a las características socioculturales, pero sí dependen del currículo implementado. Esta diferencia en el logro académico en Español y en Matemáticas, dependiendo del contexto de rigen de los alumnos, ha sido reportado por Bautista (2012), quién encontró 
que el contexto de procedencia (condiciones sociales, culturales y económicas de los centros escolares) tiene un efecto significativo en el logro académico de estudiantes mexicanos del Sureste, de manera más alta y marcada en la asignatura de español, comparado con los resultados en Matemáticas.

Finalmente, los hallazgos de este estudio sugieren también que los alumnos que asisten a escuelas particulares gozan de entornos que les proveen de mayores oportunidades de aprendizaje y de capital cultural, lo que redunda en sus mejores resultados en evaluaciones de logro académico (Backhoff, 2011; Backhoff et al., 2010), debido a que un mayor nivel socioeconómico provee mayores oportunidades de aprendizaje y de capital cultural, además de las oportunidades de aprendizaje propias en el salón de clases (Alanís et al., 2009; Blanco, 2008; Carvallo, 2006; Carvallo et al., 2007).

\section{Conclusiones}

Los resultados de este estudio no solo confirman los datos y hallazgos en torno a que los estudiantes que proceden de contextos sociodemográficos más desfavorecidos, principalmente de escuelas del sistema indígena y de las zonas rurales obtienen los más bajos indicadores en evaluaciones a gran escala del logro académico, mientras que los alumnos de escuelas privadas son los que obtienen mejores resultados en dichas evaluaciones. También muestran evidencias más precisas de estas diferencias en comparaciones de una evaluación a gran escala con opción de respuesta correcta única, con evaluaciones de aprendizajes esperados con respuestas jerarquizadas y a pequeña escala, pero sobretodo permiten identificar relaciones diferenciales entre tres indicadores de desempeño o logro académico.

A manera de conclusión, la evaluación de los aprendizajes esperados permite detectar de una manera más precisa las diferencias y deficiencias, vinculadas con los contextos de procedencia de los alumnos que participaron en el presente estudio.

Una limitación sustancial de este trabajo es la falta de medición y control de variables importantes como el capital cultural o el nivel socioeconómico, el clima escolar, y diversas variables que han evidenciado a lo largo de diferentes estudios, el aporte significativo que tienen en el logro académico. En estudios posteriores deberán considerarse dichas variables para presentar un modelo teórico mejor construido y que aporte más a la toma de decisiones en políticas educativas.

\section{Referencias}

Alanís, J. P., Ávila, V. y Lerma, E. (2009). Las implicaciones de la prueba ENLACE en educación primaria y su relación con el contexto socioeconómico. Revista Electrónica Praxis Investigativa Redie, 1(1), 22-33. http://redie.mx/librosyrevistas/PRAXIS\%20INVESTIGATIVA\%20ReDIE\%201.pdf

Backhoff, E. (2011). La inequidad educativa en México: diferencias en el aprendizaje de la comprensión lectora en educación básica. Profesorado, Revista de Currículum y Formación del Profesorado, 5(3), 88-102. https://recyt.fecyt.es/index.php/profesorado/article/view/41451

Backhoff, E., Sánchez, A., Peón, M. y Andrade, E. (2010). Comprensión lectora y habilidades matemáticas de estudiantes de educación básica en México: 2000-2005. Revista Electrónica de Investigación Educativa, 12(1), 1-15. https://redie.uabc.mx/redie/article/view/242

Bautista, A. K. (2012). La desigualdad social bajo la prueba ENLACE. Reencuentro: Análisis de Problemas Universitarios, 23(64), 27-45.

Bazán, A., Barrera, D. y Vega, N. (2013). Validación de constructos de competencias de lectura y producción de textos en los inicios de la generalización de la Reforma en la primaria Mexicana. Revista Iberoamericana sobre Calidad, Eficacia y Cambio en Educación, 11(4), 61-76.

https://revistas.uam.es/index.php/reice/article/view/2890 
Relaciones entre contexto de procedencia y logro académico al terminar primaria

Blanco, E. (2008). Factores escolares asociados a los aprendizajes en la educación primaria mexicana: un análisis multinivel. Revista Iberoamericana sobre Calidad, Eficacia y Cambio en Educación, 6(1), 58-84. https://revistas.uam.es/index.php/reice/article/view/5454

Carvallo, M. (2006). Factores que afectan el desempeño académico de alumnos de educación secundaria. (Tesis doctoral). Universidad de Guadalajara, México.

Carvallo, M., Caso, J. y Contreras, L. A. (2007). Estimación del efecto de variables contextuales en el logro académico de estudiantes de Baja California. Revista Electrónica de Investigación Educativa, 9(2), 1-15. https://redie.uabc.mx/redie/article/view/170

Ceylan, V. K. y Kesici, A. E. (2017). Effect of blended learning to academic achievement. Journal of Human Sciences, 14(1), 308-320. https://www.j-humansciences.com/ojs/index.php/IJHS/article/view/4141

Coleman, J. S., Campbell, E., Hobson, C., McPartland, J., Mood, A., Weinfeld, F. y York, R. (1966). Equality of educational opportunity. Oficina de impresión de los Estados Unidos.

Cornejo, R. y Redondo, J. (2007). Variables y factores asociados al aprendizaje escolar: una discusión desde la investigación actual. Estudios Pedagógicos, 33(2), 155-175. https://doi.org/10.4067/S071807052007000200009

Emory, R., Caughy, M., Harris, T. R. y Franzini, L. (2008). Neighborhood social processes and academic achievement in elementary school. Journal of Community Psychology, 36(7), 885-898.

https://doi.org/10.1002/icop.20266

Evaluación Nacional del Logro Académico en Centros Escolares. (2013). Resultados Históricos Nacionales 2006-2013. https://www.inee.edu.mx/wp-content/uploads/2019/05/Resultados hist nac 2006 2013.pdf

Ferrari, A. M. (2014). Validación de instrumentos de evaluación por competencias en Matemáticas y Español en sexto grado de primaria en Morelos y Sonora (Tesis de licenciatura). Universidad Autónoma del Estado de Morelos, México.

González-Pienda, J. A. (2003). El rendimiento escolar. Un análisis de las variables que lo condicionan. Revista Galego-Portuguesa de Psicoloxia e Educación, 7(8), 240-247. https://core.ac.uk/reader/61900315

Gutiérrez, H., Aguiar, M. E., Gutiérrez, P. y Garibay, C. (2015). Resultados en matemáticas en enseñanza media superior: ENLACE Jalisco y factores asociados. Revista de Educación y Desarrollo, 35, 42-51. http://www.cucs.udg.mx/revistas/edu desarrollo/anteriores/35/35 Gutierrez.pdf

Hernández-Padilla, E. y Bazán, A. (2016). Efectos contextuales, socioeconómicos y culturales, sobre los resultados de México en Lectura en PISA 2009. Revista Iberoamericana sobre Calidad, Eficacia y Cambio en Educación, 14(2), 79-95. https://revistas.uam.es/index.php/reice/article/view/2743

Hernández-Padilla, E. y González-Montesinos, M. J. (2011). Modelo de ecuación estructural que evalúa las relaciones entre el estatus cultural y económico del estudiante y el logro educativo. Revista Electrónica de Investigación Educativa, 13(2), 188-203. https://redie.uabc.mx/redie/article/view/290

Instituto Nacional para la Evaluación de la Educación. (2013). México en PISA 2012. Autor.

Instituto Nacional para la Evaluación de la Educación. (2014). El aprendizaje en preescolar en México Informe de resultados EXCALE 00. Aplicación 2011: Lenguaje y comunicación y Pensamiento matemático. Autor.

Instituto Nacional para la Evaluación de la Educación (2016a). El aprendizaje en tercero de secundaria en México. Informe de resultados EXCALE 09. Aplicación 2012: Español, Matemáticas, Ciencias y Formación 
Cívica y Ética. Autor.

Instituto Nacional para la Evaluación de la Educación (2016b). México en PISA 2015. Autor.

Instituto Nacional para la Evaluación de la Educación (2016c). Resultados Nacionales del Tercer Estudio Regional Comparativo y Explicativo TERCE 2013. Autor.

Instituto Nacional para la Evaluación de la Educación (2016d). Resultados nacionales 2015, Matemáticas. http://www.inee.edu.mx/images/stories/2016/planea/Planea10.pdf

Jensen, B., Pérez-Martínez, M. G. y Aguilar-Escobar, A. (2015). Framing and assessing classroom opportunity to learn: the case of Mexico. Assessment in Education: Principles, Policy \& Practice, 23(1), 149-172. https://doi.org/10.1080/0969594X.2015.1111192

Salazar, R., Flores, U., Florez, N., Luna, M. y Valenti, G. (2010). Desempeño escolar México 2010. Un enfoque en la calidad con equidad. Reporte de investigación. FLACSO.

http://www.enlace.sep.gob.mx/content/ms/docs/2013/DESEMPENO ESCOLAR MEXICO 2010.pdf

Secretaría de Educación Pública (2011). Plan de estudios de la SEP 2011. Autor.

Steinmayr, R., Dinger, F. C. y Spinath, B. (2011). Motivation as a mediator of social disparities in academic achievement. European Journal of Personality, 26(3), 335-349. https://doi.org/10.1002/per.842

Tapia, L. A. y Valenti, G. (2016). Desigualdad educativa y desigualdad social en México. Nuevas evidencias desde las primarias generales en los estados. Perfiles educativos, 38(151), 32-54.

https://doi.org/10.22201/iisue.24486167e.2016.151.54885 